\title{
Medicinal Chemistry as Foundation for Contraception, Birth Control, Family Planning and Fertility
}

Kraetschmer $\mathbf{K}^{*}$

Austrian American Medical Research Institute, Hermanng 4, A-2700 WrNeustadt, Austria

\begin{abstract}
Contraception plays a pivotal role in birth control and family planning and thus affects millions of women not only in the U.S. but also world-wide. Efforts are made in the U.S. to reduce the percentage of unwanted pregnancies, i.e., $49 \%$, which compares unfavorably with Western Europe's percentage of $34 \%[1,2]$. Despite a vast and steadily-increasing literature on the issues of family planning and birth control, several questions are still awaiting clarification, especially with regard to medicinal chemistry, i.e., effects of chemical substances on human endocrinology. The following analysis aims at identifying problem areas in contemporary research and draws attention to flawed data in scholarly publications.
\end{abstract}

\section{Hormones: Endocrinology and Chemistry}

The menstrual cycle of the female reproductive system is primarily under the control of the hypothalamic Gonadotropin Releasing Hormon $(\mathrm{GnRH})$ and the hormons released by the anterior pituitary, i.e., Luteinizing Hormon ( $\mathrm{LH}$ ) and Follicle Stimulating Hormon (FH). The uterine cycle is divided into a proliferative phase and a secretory or luteal phase. The proliferative phase, also called preovulatory or follicular, is under the influence of estrogens stemming from the developing follicle and lasts from the 5th to the 14th day of the menstrual cycle. The secretory phase is under the influence of estrogen and progesterone from the corpus luteum and lasts from the 15th day to the 28th day. Late in the luteal phase the anterior pituitary and the endometrium produce prolaktin, whose function is not fully understood. The ovarian steroids like cholesterol, bile acids, and vitaminD contain the cyclopentanoperhydrophenanthrene nucleus. Figures 1 and 2 shows the biosynthesis and metabolism of estrogens as well as the precursor steroids Cholesterol and Pregnenolone. Ovarian Hormones: 17beta-estradiol, estrone, and estriol.

The naturally occurring estrogens secreted by the theca interna and granulosa cells of the ovarian follicles, the corpus luteum, and the placenta are the following $\mathrm{C}_{18}$ steroids: 17beta-estradiol, estrone, and estriol. These steroids do not have an angular methyl group attached to the 10 position or a Delta -3-keto configuration in the A ring $[3,4]$.

In the biosynthetic pathway they are formed from androgens, but they are also formed in the circulation by aromatization of androstenedione. The enzyme aromatase catalyzes both, the conversion of androstenedione to estrone and the conversion of testosterone to 17beta-estradiol (E2) [2]. 17beta-estradiol (E2), the major secreted estrogen, is in equilibrium in the circulation with estrone. Estrone ist metabolized to estriol, probably primarily in the liver. Estradiol is the most potent and estriol the least potent of the three estrogens.

Estrogens: secretion and metabolism. The concentration of estradiol in the plasma during the menstrual cycle varies and reaches a first peak of approximately $200 \mathrm{pg} / \mathrm{mL}$ around day 13 , i.e., just before ovulation, and a second peak of about $110 \mathrm{pg} / \mathrm{mL}$ around day 19-22, i.e., during the midlutel phase. Almost all of this estradiol stems from the ovary. The estradiol secretion rate is $36 \mathrm{ug} / \mathrm{d}(133 \mathrm{umol} / \mathrm{d})$ in the early follicular phase, $380 \mathrm{ug} / \mathrm{d}$ just before ovulation, and $250 \mathrm{ug} / \mathrm{d}$ during the midluteal phase. Following menopause, estrogen secretion declines and stays at low levels. In the liver, estrogens are oxidized or converted to glucuronide and sulfate conjugates. Considerable amounts are secreted in the bile and reabsorbed into the blood stream (enterohepatic circulation). At least 10 different metabolites of estradiol can be found in the human urine [2].

Progesterone is $\mathrm{a}_{21}$ steroid secreted by the corpus luteum, the placenta, and-in small amounts-by the follicle. 17alphaHydroxyprogesterone is seemingly secreted along with estrogens from the ovarian follicle, and its secretion parallels that of 17beta-estradiol. Progesterone has a short half-life, and in the liver it is is converted to pregnanediol. Pregnanediol is conjugated to glucuronic acid and excreted in the urine. The plasma progesterone level in women is approximately $0.9 \mathrm{ng} / \mathrm{mL}$ ( $3 \mathrm{nmol} / \mathrm{L}$ ) during the follicular phase of the menstrual cycle. During the luteal phase, large amounts of progesterone are produced by the corpus luteum, and ovarian secretion increases about 20 -fold. The resulting increase in plasma progesterone leads to a peak value of approximately $18 \mathrm{ng} / \mathrm{mL}(60 \mathrm{nmol} / \mathrm{L})$.

\section{The Effects of Hormones and Contraceptive Methods based on Cyclic Changes}

Hormones have effects on various organs: endocrine organs (hypothalamus and pituitary), the breasts, and especially the female genitalia, i.e., ovarian follicles and uterine tubes, uterine muscle, uterine blood flow, endometrium, cervix, and vagina. The cervix of the uterus, although continuous with the body of the uterus, differs from it in a number of ways, above all through regular changes in the cervical mucus; cyclic desquamations occurring in the corpus of the uterus are absent in the cervix. Estrogen makes the cervical mucus thinner and more alkaline promoting in this way the survival and transport of sperms. Progesterone, on the other hand, makes it thick, tenacious and cellular. "The mucus is thinnest at the time of ovulation, and its elasticity, or spinnbarkeit, increases so that by mid-cycle, a drop can be stretched into a long, thin thread that may be $8-12 \mathrm{~cm}$ or more in length. In addition, it dries in an arborizing, fernlike pattern" [2].

*Corresponding author: Kraetschmer K, Austrian American Medical Research Institute, Hermanng 4, A-2700 WrNeustadt, Austria, Tel: +12128561075; E-mail: kurt.kraetschmer@aon.at

Received May 30, 2017; Accepted June 14, 2017; Published June 18, 2017

Citation: Kraetschmer K (2017) Medicinal Chemistry as Foundation for Contraception, Birth Control, Family Planning and Fertility. Med Chem (Los Angeles) 7: 188-192. doi: 10.4172/2161-0444.1000455

Copyright: $\odot 2017$ Kraetschmer K. This is an open-access article distributed under the terms of the Creative Commons Attribution License, which permits unrestricted use, distribution, and reproduction in any medium, provided the original author and source are credited. 


\author{
Cholesterol $>$ Pregnenolone $>$ 17alpha-Hydroxypregnenolone $>$ Dehydroepiandrosterone $>$ Androstenedione \\ $><$ Testosterone
}

Testosterone --Aromatase-- > 17beta-Estradiol (E2) > other metabolites

\author{
Androstenedione $>$--Aromatase-- > Estrone (E1)
}

Estrone > -( in the liver )- >16-Ketoestrone > 16alpha-Hydroxyestrone > Estriol

Figure 1: Biosynthesis and metabolism of estrogens and precursor steroids [2].

Cholesterol $>$ Pregnenolone $>$ - 3beta-Hydroxysteroiddehydrogenase- $>$ Progesterone $>$ Pregnanediol $>$ Sodium pregnanediol-20-glucuronide

\author{
Progesterone $>-17$ a-Hydroxylase(P450c17)->17alpha-Hydroxyprogesterone $\quad>\quad-\quad 17,20$ Lyase- > \\ Androstenedione
}

Figure 2: Progesterone: secretion and metabolism.

Ovulation occurs at about the 14th day of the cycle where the distended follicle ruptures, and the ovum is extruded into the abdominal cavity. The ovum is then picked up by the fimbriated ends of the uterine tubes (oviducts), transported to the uterus, and -- unless fertilization occurs expelled through the vagina. The process of ovulation is associated with the typical cyclic changes in plasma concentration of hormones such as progesterone, 17alpha-Hydroxypogesterone, 17beta-Estradiol, and the gonadotropins secreted by the anterior pituitary, i.e., luteinizing hormone and follicle stimulating hormone, as well as inhibin (a factor of testicular origin that inhibits FSH secretion). Besides these indicators of ovulation, changes in basal body temperature are particularly noteworthy. "A convenient and reasonably reliable indicator of the time of ovulation is a change-usually a rise-in the basal body temperature. The cause of the temperature change at the time of ovulation is probably the increase in progesterone secretion, since progesterone is thermogenic" [2].

Cyclic changes have been of particular interest to investigators who described the so-called non-hormonal methods of contraception. The above mentioned change in basal body temperature is the basis for the so-called "Body Temperature method" (BBT) described for the first time by Van de Velde in 1927 [4]. 24 hours to 36 hours following ovulation the temperature rises on 3 subsequent days by at least $0.2^{\circ} \mathrm{Celsius}$, and measurement of this rise in temperature is used to determine the beginning of the infertile phase. According to the World Health Organization (WHO), this process is defined as a rise of at least $0.2^{\circ} \mathrm{Celsius}$ (compared to the temperature during the preceding 6 days) occurring within 48 hours maximum, and lasting at least 3 days [4]. Qualitative and quantitative changes in the cervical mucus are the basis for the so-called "Billings ovulation" or "cervical mucus method" described by the Australian neurologist John Billings in 1964. As the changes in cervical mucus structure indicate the beginning of the fertile phase, they are also used for the diagnosis of sterility. In contemporary research on contraceptive technology, the evaluation of cervical mucus is the basis for the so-called "Ovulation" and "TwoDay" methods [5].
According to this research, their efficacy in case of perfect use, i.e., $3 \%$ and $4 \%$ respectively, is superior to female condom (5\% without spermicide) and diaphragm ( $6 \%$ with spermicidal cream or jelly).

A combination of basal body temperature and cervical mucus is the "symptothermal" method, described by Rötzer in 1968, which also recommends observation of symptoms such as mastalgia and "mittelschmerz". Generally, it is considered as the most effective of the so-called "fertility awareness-based" methods due to a perfect use failure rate of $0.4 \%$. It is described by contraceptive technology as a "double-check" method, "based on evaluation of cervical mucus to determine the first fertile day and evaluation of cervical mucus and temperature to determine the last fertile day" [5]. The oldest of the natural family planning methods is the calendar method described by Knaus and Ogino between 1932-1933. Even older is the "lactational amenorrhea method" (LAM). It is based on the effects of prolactin on the hypothalamus. Nursing has long been known to be an important method of birth control, and contemporary research considers LAM as "a highly effective, temporary method of contraception" [5]. Nursing stimulates prolactin secretion, and prolactin inhibits hypothalamic Gonadotropin Releasing Hormone ( $\mathrm{GnRH}$ ) secretion. As a consequence, the action of GnRH on the pituitary is inhibited and the action of gonadotropins on the ovaries is antagonized. "Ovulation is inhibited, and the ovaries are inactive, so estrogen and progesterone output falls to low levels. Consequently, only $5-10 \%$ of women become pregnant again during the suckling period“ [2].

The five methods mentioned above, i.e., basal body temperature, ovulation, symptothermal, calendar and lactational amenorrhea are counted among the non-hormonal methods, and the first four of them are frequently classified as fertility awareness or natural family planning. In contrast to other methods of contraception, these methods do not require any drugs or devices, advantages which have been underscored also by the American Congress of Obstetricians and Gynecologists (ACOG): 
"They cost very little Many women like the fact that fertility awareness is a form of birth control that does not involve the use of medications or devices" [6]. With respect to efficacy, the ACOG states that "fewer than 1-5 women out of 100 " will get pregnant during the first year of perfect use. Efficacy of contraceptive methods is, in fact, a highly important issue given that in the U.S. the percentage of pregnancy that are unwanted (49\%) is considerably higher than the percentage in other countries (34\% in Western Europe) [1]. In light of such data, efforts are being made by some U.S. organizations to decrease the number of pregnancies by suggesting the use of certain methods of birth control, especially Long Acting Reversible Contraceptive (LARC) methods which are hailed as "the most highly effective" methods [1]. However, accurate information on the efficacy of these contraceptive methods is difficult to obtain, and ratings according to efficacy are controversial.

\section{Tables, Surveys and Ratings of Contraceptive Methods}

The most reliable authority on issues of contraceptive efficacy, contraceptive technology research, presented an overview of methods as early as 2011, distinguishing between perfect use and typical use and differentiating also between "first year of use" and "continuing use at one year" [5]. A summary the methods including their estimates is available in form of a "Contraceptive Failure Table." According to this table, the Long Acting Reversible Contraceptives, i.e., Implants and Intrauterine Devices, appear as the most effective, especially the implant Implanon (precursor of Nexplanon) with a failure rate of 0.05 for both perfect and typical use. Among intrauterine devices, Mirena (LNg) with a perfect and typical use failure rate of 0.2 is superior to ParaGard (copper T) with a perfect use failure rate of 0.6 and a typical use failure rate of 0.8 . About equally effective are Depo-Provera with 0.2 perfect use ( 6 typical use), NuvaRing 0.3 perfect use ( 9 typical use), Evra patch 0.3 perfect use ( 9 typical use), as well as combined pill and progestin-only pill 0.3 perfect use (9 typical use). Among the so-called "fertility awarenessbased" methods, whose typical use failure rate of 24 is based on obsolete data from 1995, $[5,6]$ the symptothermal methods with a perfect use failure rate of 0.4 appears almost equally effective as pill and progestinonly pill (0.3), Evra patch (0.3), and NuvaRing (0.3) are more effective as ParaGard (copper $\mathrm{T}$ ) with a perfect use failure rate of 0.6 . The ovulation method with a perfect use failure rate of 3 is almost as effective as male condom without spermicide ( 2 perfect use) but superior to female condom without spermicide ( 5 perfect use). The TwoDay method with a perfect use failure rate of 4 equals coitus interruptus (4 perfect use), and the Standard Days method with a perfect use failure rate of 5 is still superior to diaphragm (with spermicidal cream or jelly) with a perfect use failure rate of 6

As mentioned above, the symptothermal method with a perfect use failure rate of 0.4 is based on evaluation of cervical mucus to determine the first fertile day and on evaluation of cervical mucus as well as temperature to determine the last fertile day [4]. The two methods based on the evaluation of cervical mucus, i.e., Ovulation and TwoDay, have perfect use failure rates of 3 and 4 respectively, and the Standard Days method which avoids intercourse on cycle day 8 through 19 has a failure rate of 5 . Among the definitive methods, male sterilization with a perfect use failure rate of 0.10 (typical use 0.15 ) is superior to female sterilization with 0.5 for both perfect and typical use.

Concerning Emergency contraception, i.e., pills or insertion of a copper intrauterine contraceptive following unprotected intercourse, contraceptive technology claims that they substantially reduce the risk of pregnancy. The only dedicated products marketed specifically for emergency contraception are Ella, Plan B One-Step, and Next Choice. Lactational Amenorrhea method (LAM) is considered to be a remarkably effective though only temporary method of contraception, and another method of contraception must be implemented for effective protection against pregnancy, as soon as one of the following conditions arises: menstruation resumes, the frequency or duration of breastfeeds is reduced, bottle feeds are introduced, or the baby reaches 6 months of age.

These estimates presented by contraceptive technology research in 2011 are based on data for the U.S. and converge only partially with data provided by international research. German researcher published data on contraceptive methods as early as 2000 [4]. In the context of a chronological study of the phenomenon of contraception in the history of medicine 15 different methods are being highlighted under the traditional terminology and ranked according to the Pearl-Index (number of unwanted pregnancies per 100 women years or 1200 months of application). This ranking shows "tubal sterilization" (Pearl index 0.09-0.4) together with "depot-gestagens" (Pearl index 0.03-0.9), as the most efficacious, followed by "monophasic combined pill" $(0.1$ $1.0)$, "oral hormonal sequential contraceptives" $(0.2-1.4)$, "minipill" (1), "intrauterine pessary" (0.14-2) and the symptothermal method (0.8) [4]. Concerning the other natural family planning methods, "basal temperature" (Pearl index of 1-3) seems comparable to "diaphragm and spermicide" (Pearl index 2-4) or "condom" (4-5), while "cervical mucus" (15-32) and "calendar" (15-40) roughly approximate the efficacy of "chemical spermicides" (12-20) or "coitus interruptus" (8-38).

Due to the Pearl index of 0.8 , the symptothermal method was recognized by German research as the most effective of the natural family planning methods and considered to be one of the "safe contraceptive methods" [4], notwithstanding the problem of irregular cycles, which restricts substantially the use of this method and necessitates the additional use of other methods. Numerous other ratings and surveys have been proposed, but many of them lack both completeness and accuracy. Thus, the FDA presents a consumer-friendly survey of FDA-approved methods, [7] which uses as its source contraceptive technology but omits some of the internationally recognized methods listed in the Contraceptive Technology Failure Table [5]. Another highly influential organization, the Center for Desease Control [8] presents a ranking which shows the fertility awareness-based methods as the least effective due to a failure rate of $24 \%$, an estimate which is based, alas, on obsolete data from the last century [5]. What must be borne in mind also in evaluating the accuracy of data presented in the various surveys and rankings is the fact that they focus almost exclusively on efficacy, and there is no ranking available that takes into account both crucial variables, i.e., efficacy plus safety. Although some publications make reference to the issue of safety by mentioning medical eligibility criteria, adverse events, side effects, risks, and complications, their primary goal seems to be emphasis on efficacy, as can be seen from publications on LARCs [1] or on implantable contraception [8].

As these studies do not offer an in-depth analysis of adverse events, side effects, risks, and complications, they stand in contrast to international research where side effects, interactions, contraindications, and also forensic ramifications are discussed exhaustively [4]. In order to appreciate the complexity of adverse events associated with the use of both, implants and intrauterine devices a detailed analysis of the mechanism of action of some of the most frequently used LARCs seems in place $[1,9]$.

\section{Adverse Events, Side Effects, Risks, Contraindications, and Complications of Contraceptive Methods}

Implants are are available in the form of one or more sub-dermally 
placed rods that slowly release progestin, whereby these sustainedrelease systems rely on simple diffusion of steroid hormones through semipermeable plastics. "The synthetic progestin passes from the plastic into the surrounding tissues and enters the circulatory system through absorption by the local capillary network. The release rate of the progestin depends on the surface area and the density of the plastic (silastic or ethylene vinyl acetate) in which the progestin is contained" [9].

In the case of one of the frequently used implants, each Jadelle rod contains $75 \mathrm{mg}$ of levonorgestrel for a total of $150 \mathrm{mg}$. The thin, flexible Jadelle rods are wrapped in silastic tubing, $43 \mathrm{~mm}$ in length and 2.5 $\mathrm{mm}$ in diameter. In contrast to Norplant, the levonorgestrel is packed into the capsules in crystal form, and the core of the Jadelle rod is a mixture of levonorgestrel and an elastic polymer (dimethylsiloxane/ methylvinylsiloxane). During the first 6-12 months of use, Jadelle as well as Norplant releases a total of about $80 \mathrm{mg}$ of levonorgestrel every 24 hours, giving a plasma concentration of $0.35 \mathrm{ng} / \mathrm{mL}$. Subsequent to the first year, the release rate gradually declines to a relatively constant rate of $30-35 \mathrm{mg} /$ day. At 5 years, the overall release rate is $25 \mathrm{mg} /$ day, with corresponding levonorgestrel plasma concentration of 0.25-0.35 ng/ $\mathrm{mL}$. For the purpose of comparison, progestin-only oral contraceptive pills too deliver about $80 \mathrm{mg}$ of levonorgestrel per day; combined oral contraceptives with levonorgestrel as the active progestin deliver 50-125 $\mathrm{mg}$. Peak serum levels after ingestion of $75 \mathrm{mg}$ of levonorgestrel reach 1.5-2.0 ng/mL; after ingestion of $150 \mathrm{mg}$ of levonorgestrel, serum peaks are at $2.7-4.2 \mathrm{ng} / \mathrm{mL}$. These serum peaks are reached from 30 minutes to 2 hours after ingestion and are followed by a rapid decline, with an average half-life of 10-12 hours. This is in contrast to the stable, low serum concentrations of progestin accomplished with the sustainedrelease systems.

The Nexplanon implant measures $40 \mathrm{~mm} \times 2.0 \mathrm{~mm}$ and consists of one nonbiodegradable rod of $40 \%$ ethylene vinyl acetate and $60 \%$ etonogestrel (the 3-keto derivative of desogestrel) and is covered with a rate-controlling ethylene vinyl acetate membrane $0.06 \mathrm{~mm}$ thick. The rod contains $68 \mathrm{mg}$ etonogestrel that is slowly released, initially at 60$70 \mu \mathrm{g} /$ day. It decreases to $35-45 \mu \mathrm{g} /$ day at the end of the first year, to $30-40 \mu \mathrm{g} /$ day at the end of the second year, and then to 25 to $30 \mu \mathrm{g} /$ day at the end of the third year. The high initial rate of absorption is apparently due to a significant amount of etonogestrel released from the uncovered ends of the implant. Peak serum concentrations of $266 \mathrm{pg} / \mathrm{mL}$ of etonogestrel are reached within one day after insertion, suppressing ovulation, which requires only 90 or more $\mathrm{pg} / \mathrm{mL}$. Serum concentrations of etonogestrel are adequate to provide contraception for 5 years, and WHO data do in fact suggest efficacy for that long.

For progestin-containing implants there are two primary mechanisms of action: inhibition of ovulation and restriction of sperm penetration through cervical mucus. Antiestrogenic actions of the progestins affect the cervical mucus, making it viscous, scanty, and impenetrable to sperm, inhibiting in this way fertilization. At high doses, progestins also inhibit pituitary gonadotropin secretion of luteinizing hormone ( $\mathrm{LH}$ )and follicle-stimulating hormone (FSH), inhibiting thereby follicular maturation and ovulation. This dual effect permits contraceptive efficacy to be maintained even though ovulation is not consistently inhibited in etonogestrel implant users toward the end of the 3-year period of use. "Even if follicles grow during use of progestin implants, oocytes are not fertilized. If the follicle ruptures, the abnormalities of the ovulatory process prevent release of a viable egg. Although progestins suppress endometrial activity, which makes the endometrium unreceptive to implantation, this is not a contraceptively important effect since the major mechanisms of action prevent fertilization" [9].
Besides implants, intrauterine devices (IUDs) are considered as the most efficacious methods of contraception. They are either coppercontaining or levonorgestrel-releasing. The copper-containing IUD, ParaGard, is a nonhormonal device containing $380 \mathrm{~mm}^{2}$ of copper around the arms and stem. "The four levonorgestrel-releasing IUDs (LNG-IUDs) include two devices that contain $52 \mathrm{mg}$ of levonorgestrel (Mirena and Liletta), one device that contains $19.5 \mathrm{mg}$ (Kyleena), and a slightly smaller device that contains $13.5 \mathrm{mg}$ (Skyla)" [1]. Regarding the mechanisms of action of the IUDs it is claimed that "IUDs do not cause the destruction of an implanted embryo but rather work primarily by preventing fertilization. The copper-containing IUD releases copper ions that are toxic to sperm. The LNG-IUD inhibits ovulation and thickens cervical mucus, which obstructs the penetration of sperm" [1].

From an economic viewpoint it is understandable that proponents of LARCs receiving financial support from pharmaceutical companies are inclined to claim that "almost all women can safely use IUDs" [1]. From a strictly medical perspective, however, there is an ethical responsibility to draw attention to well-known adverse events, as has been done by physiologists: "Although the mechanism of action of IUDs is still unsettled, there is evidence that at least those containing copper exert a spermicidal action. Their usefulness is limited by their tendency to cause intrauterine infections" [2]. Pelvic inflammatory disease is in fact a well-known hazard, and even proponents of IUDs do admit that there exists quite a number of conditions which preclude the use of IUDs, as for example hypersensitivity to copper or other components: "women who have hypersensitivity to copper, which would preclude the use of the copper-containing IUD, or hypersensitivity to other components of either type of IUD; women with a current pelvic infection or a sexually transmitted disease (STD); women with gynecologic cancers; and women with certain other serious medical conditions Women who have current purulent cervicitis or known chlamydial infection or gonococcal infection should not undergo insertion of an IUD“ [1]. There are altogether 15 conditions for which at least one LARC method should not be used or should generally not be used, according to the Medical Eligibility Criteria (MEC) for Initiation of LARC Methods [1]. It must be borne in mind that these conditions which preclude the use of a device must be distinguished from conditions which emerge as adverse events, once the device has been implanted; these again must be distinguished from complications that can occur during the implantation or the removal, both of which can require surgical interventions.

Given a wide array of adverse events, it is understandable that the side effects of all forms of LARCs are of general interest. At present, information is readily available through several websites on specific substances, such as the one on medroxyprogesterone-acetate [10] or on implants in general [11]. These and other websites provide information on well-known side effects and risks such as menstrual bleeding changes, reduction in bone mineral density, cardiovascular and thromboembolic risk, amenorrhea, unscheduled bleeding, headaches, acne, nausea, mood changes, loss of libido, etc. Only sporadically less-known events are reported such as "lost" rods and perforation of uterine wall with subsequent dislodgement of the device in the abdominal cavity. Almost unmentioned go some systemic effects of hormons such as cholelithiasis resulting from the production of lithogenic (cholesterol-rich) liver-bile with reduced content of lecithin and cholic acid [12].

\section{Conclusion}

In the face of the numerous adverse events, risks, and complications associated not only with the use but also with the implantation and the removal of devices the question arises as to how to define "safety" of 
contraceptive methods -- in addition to protection against sexually transmitted diseases. Frequently claims are being made to the effect that LARCs and other hormonal methods can be used safely: "Almost all women can safely use IUDs" [1]. In view of those women who consider unsafe any drug or device that can cause such serious conditions as hemorrhagic bleeding or pelvic inflammatory disease it must be stated that the concept of "safety" is frequently used in a misleading fashion. It seems necessary, therefore, to determine as to whether it is ethically and medically correct to call a device safe if it has the potential of affecting adversely a woman's health. As the term "safe" is used nonchalantly in some instances, it must be suggested that future research investigate not only singular adverse events in the use of contraceptive methods but integrate these events into the larger context of quality of life [13]. For this purpose, a common terminology criteria for adverse events could be formulated following the instruments developed by the National Cancer Institue [14].

Also, to better understand the mechanisms of action of implants and devices, especially in the context of pharmakogenetics, cooperative research projects seem desirable with contributions from chemists, biologists, physiologists and endocrinologists. Information gained from such investigations must be passed on to the consumer in an impartial fashion to ascertain each woman's autonomous decision-making process in matters of contraception. Such striving for completeness of information is not only an act of courtesy vis-a-vis the patient but an ethical obligation according to the principle of informed consent. This principle, based on the bill of rights formulated by the American Hospital Association as early as 1973 [15] is internationally honored as an ethical imperative and should be an integral part of any doctorpatient interaction in the 21 st century.

\section{References}

1. Curtis KM, Peipert JF (2017) Long-Acting Reversible Contraception. New England Journal of Medicine 376: 461-468.

2. Ganong WF (1995) Review of Medical Physiology. 17th edn. Prentice-Hall International Inc., East Norwalk, Connecticut, USA.

3. Norman AW, Henry HL (2014) Hormones. 3rd edn. Academic Press.

4. Gröger S, Grüne B (2000) Kontrazeption. In: Diedrich K (ed.), Gynäkologie und Geburtshilfe. Berlin: Springer. pp: 60-87.

5. Trussell J (2011) Contraceptive Efficacy. In: Hatcher RA, Trussell J, Nelson AL Cates W, Kowal D, Policar M. Contraceptive Technology. Twentieth Revised Edition. Ardent Media, New York, NY, USA, pp: 3-32.

6. American Congress of Obstetricians and Gynecologists FAQ. Available from www.acog.org/Patients/FAQs/Fertility-Awareness-Based-Methods-of-FamilyPlanning (Accessed Feb 14, 2017).

7. Food and Drug Administration. Accessed January 16, 2017.

8. Center for Disease Control and Prevention. US Medical Eligibility Criteria for Contraceptive Use 2016 (Accessed March 26, 2017).

9. French V, Darney P (2016) Glob Libr. Women's Med.

10. Medroxyprogesterone-acetate (Accessed 6 May, 2017).

11. Implants. NHS United Kingdom (Accessed 6 May 2017).

12. Zink C (1990) Pschyrembel Klinisches Wörterbuch. Berlin, New York: Walter de Gruyter, p: 558.

13. Zethraeus N, Dreber A, Ranehill E, Blomberg L, Labrie F, et al. (2017) A firstchoice combined oral contraceptive influences general well-being in healthy women: a double-blind, randomized, placebo-controlled trial. Fertility and Sterility 107: 1238-1245.

14. Dueck AC, Mendoza TR, Mitchell SA, Reeve BB, Castro KM, et al. (2015) Validity and reliability of the US National Cancer Institute's patient-reported outcomes version of the common terminology criteria for adverse events (PROCTCAE). JAMA Oncology 1: 1051-1059.

15. Schott H (1993) Die Chronik der Medizin. Dortmund: Harenberg Verlag, p: 620. 